\title{
Pharmacokinetics and metabolism of I3-cis-retinoic acid (isotretinoin) in children with high-risk neuroblastoma - a study of the United Kingdom Children's Cancer Study Group
}

\section{GJ Veal', M Cole', J Errington', ADJ Pearson², ABM Foot ${ }^{3}$, G Whyman ${ }^{4}$ and AV Boddy*,' on behalf of the UKCCSG Pharmacology Working Group}

'Northern Institute for Cancer Research, University of Newcastle upon Tyne, Newcastle upon Tyne NE2 4HH, UK; ${ }^{2}$ Royal Marsden Hospital, Surrey SM2 5PT, UK; ${ }^{3}$ Bristol Royal Hospital for Children, Bristol BS2 8BJ, UK; ${ }^{4}$ UKCCSG, University of Leicester, Leicester LEI 6TH, UK

\begin{abstract}
The administration of 13-cis-retinoic acid (I3-cisRA), following myeloablative therapy improves 3-year event-free survival rates in children with high-risk neuroblastoma. This study aimed to determine the degree of inter-patient pharmacokinetic variation and extent of metabolism in children treated with 13-cisRA. 13-cis-retinoic acid ( $80 \mathrm{mg} \mathrm{m}^{-2}$ b.d.) was administered orally and plasma concentrations of parent drug and metabolites determined on days 1 and 14 of courses 2, 4 and 6 of treatment. Twenty-eight children were studied. The pharmacokinetics of 13-cisRA were best described by a modified one-compartment, zero-order absorption model combined with lag time. Mean population pharmacokinetic parameters included an apparent clearance of $15.9 \mathrm{I} \mathrm{h}^{-1}$, apparent volume of distribution of $85 \mathrm{I}$ and absorption lag time of $40 \mathrm{~min}$ with a large inter-individual variability associated with all parameters (coefficients of variation greater than 50\%). Day I peak I3-cisRA levels and exposure (AUC) were correlated with method of administration $(P<0.02)$, with 2.44- and 1.95-fold higher parameter values respectively, when I3-cisRA capsules were swallowed as opposed to being opened and the contents mixed with food before administration. Extensive accumulation of 4-oxo-13-cisRA occurred during each course of treatment with plasma concentrations (mean \pm s.d. 4.67 $\pm 3.17 \mu \mathrm{M}$ ) higher than those of I 3-cisRA $(2.83 \pm 1.44 \mu \mathrm{M})$ in 16 out of 23 patients on day 14 of course 2. Extensive metabolism to 4-oxo- I 3-cisRA may influence pharmacological activity of 13 -cisRA.

British Journal of Cancer (2007) 96, 424-43I. doi: I0.1038/sj.bjc.6603554 www.bjcancer.com

Published online 16 January 2007

(c) 2007 Cancer Research UK
\end{abstract}

Keywords: 13-cis-retinoic acid; isotretinoin; clinical pharmacology; dosing; drug metabolism

The retinol derivative 13-cis-retinoic acid (13-cisRA) is now an established component of the treatment of high-risk neuroblastoma, despite the fact that early phase II trials conducted with low-dose 13-cisRA showed limited clinical benefit in patients with recurrent disease (Reynolds et al, 1991; Finklestein et al, 1992; Kohler et al, 2000). When 13-cisRA was administered as a highdose $\left(160 \mathrm{mg} \mathrm{m}^{-2} \mathrm{day}^{-1}\right)$, intermittent regimen in a Children's Cancer Group phase III randomised trial, a significant improvement in 3-year event-free survival (EFS) was observed (Matthay et al, 1999). Factors that may explain the efficacy observed in the latter study include the higher dose administered and the use of an intermittent dosing regimen, incorporating 2-weeks of 13-cisRA followed by a 2 week rest period, on each course of treatment (Matthay and Reynolds, 2000).

Retinoids are susceptible to oxidative metabolism, and the extensive metabolism of all-trans-retinoic acid (ATRA) in acute promyelocytic leukaemia (APL) may influence relapse rates (Muindi et al, 1992). Analysis of patient samples from a phase I study of 13-cisRA previously suggested increasing levels of the

*Correspondence: Dr AV Boddy; E-mail: Alan.Boddy@ncl.ac.uk Received 31 July 2006; revised 19 September 2006; accepted 27 November 2006; published online 16 January 2007 metabolite 4-oxo-13-cisRA during a course of treatment, although actual concentrations were not quantified (Khan et al, 1996).

An additional concern with the administration of 13-cisRA is that many neuroblastoma patients are very young. Owing to the large size and number of 13-cisRA capsules required to obtain the specified dose, younger children are physically unable to take the drug unless the capsules are opened and the contents mixed with food before administration. This practice raises concerns regarding the actual dose of drug that these patients are receiving, in addition to the possibility that the drug may be unstable during this procedure.

The current study was designed to determine the pharmacokinetics of 13-cisRA and the extent of oxidative metabolism when administered to high-risk neuroblastoma patients using an intermittent dosing regimen. In addition, the absorption of 13cisRA when administered following opening of the capsules and mixing the contents with food was investigated.

\section{MATERIALS AND METHODS}

\section{Patient eligibility and treatment}

The study protocol was approved by the UK Trent Multicentre Research Ethics Committee and written informed consent was 
obtained from patients or parents as appropriate. Patients less than 18 years with a central venous catheter, who were receiving 13-cisRA as part of their standard clinical treatment, were eligible.

13-cis-retinoic acid ( $80 \mathrm{mg} \mathrm{m}^{-2}$ b.d.) was administered orally as part of a protocol for high-risk neuroblastoma (Matthay et al, 1999), starting between 80 and 120 days post-myeloablative and radiation therapy in all cases. Drug was administered for 14 days, with a 14 day break before the next course. Toxicity was assessed by the National Cancer Institute Common Toxicity Criteria (CTC version 2.0) and patients were followed clinically on a 6-monthly basis. In patients who were unable to swallow the capsules, each capsule was snipped with a pair of scissors and the contents extruded into ice-cream or yoghourt before ingestion. Patients were not fasted before administration. On each day of the study, the administration of the studied dose of 13-cisRA was performed in hospital, supervised by a trained research nurse and fully documented.

\section{Patient details}

Age and weight together with 13-cisRA administration details were recorded for each patient. Glomerular filtration rate (GFR) was estimated according to the equation:

GFR $(\mathrm{ml} / \mathrm{min})=36.76+1.91^{\star}$ weight $(\mathrm{kg})-0.47^{\star}$ serum creatinine $\left(\mu \mathrm{moll}^{-1}\right)$ (Cole et al, 2004).

The most recent ALT, bilirubin and creatinine measurements before 13-cisRA treatment were obtained from the patients' notes (mean 26 days before administration, maximum 63 days prior).

\section{Blood sampling and analysis}

Blood samples for measurement of concentrations of individual retinoids were obtained from a central line before administration and at 1, 2, 4 and $6 \mathrm{~h}$ post-administration. Samples were obtained on days 1 and 14 (first and last day) of treatment courses 2, 4 and 6 following administration of the first dose of 13-cisRA on the particular study day. When sampling on these courses was not possible, samples were obtained on days 1 and 14 of an alternative treatment course. Course 1 was not studied in most cases to allow the families and patients to become familiar with the administration processes. Blood samples $(5 \mathrm{ml})$ were collected in heparinised tubes and centrifuged at $1200 \mathrm{~g}$ for $10 \mathrm{~min}$ at $4^{\circ} \mathrm{C}$. Plasma was separated and frozen at $-20^{\circ} \mathrm{C}$, before analysis using a highperformance liquid chromatography assay, with a limit of quantitation of $0.02 \mu \mathrm{g} \mathrm{ml}^{-1}$ for all retinoids. This analytical assay allowed for individual quantification of 13-cisRA, 9-cisRA and ATRA, in addition to the metabolite 4-oxo-13-cisRA, as previously described (Veal et al, 2002). All blood and plasma samples were wrapped in aluminium foil to protect them from light, and all sample handling was carried out in dim light. The assay was validated with regard to linearity, reproducibility and stability of the analytes according to standard practice (Shah et al, 1992).

\section{Pharmacokinetics/statistical analysis}

A population pharmacokinetic analysis was carried out using 13cisRA plasma concentrations obtained on study day 1 from the first available course of treatment for each of 28 patients (course 1 for five patients, course 2 for 20 patients and courses 3, 4 and 6 for one patient each). Peak and trough 13-cisRA levels from days 1 and 14 were determined, together with peak levels of the metabolite 4-oxo-13-cisRA on day 1 and 14; these were compared between treatment courses 2, 4 and 6 .

Using NONMEM (Beal and Sheiner), a series of population pharmacokinetic models were fitted to 13-cisRA data from the first available course of treatment, with all patients included in the analysis. A one-compartment model with first-order absorption (ADVAN2) was fitted using the FOCE estimation method with $\eta, \varepsilon$ interaction. A composite within-subject error model was used together with an exponential between-subject error model for each of the population PK parameters $\left(\mathrm{CL}, V, K_{\mathrm{a}}\right)$. Models were fitted using firstly, a diagonal, and then a full block structure for the between-subject covariance matrix. An absorption time lag was added, which was also allowed to vary across the population. A zero order absorption model with an absorption lag was also fitted (ADVAN1); both the absorption lag time and absorption duration were allowed to vary across the population.

Two further models were fitted to try to more closely model the absorption phase. The first was a 'transit model', which assumes that the absorption delay is due to the passage of drug through a chain of transit compartments (Savic et al, 2004). The number of transit compartments and the rate at which the drug moves between each of the compartments were estimated from the data. The model was parameterised in terms of CL, $V, K_{\mathrm{a}}, N$ (the number of transit compartments) and the MTT (the mean transit time to the absorption compartment). The second model was a modified zero-order absorption model with an absorption lag time. This model assumes that the appearance of the drug in a dose compartment is described by a zero-order process over a fixed duration $(D)$. Absorption into a central compartment was described by a first-order process with rate parameter $K_{\mathrm{a}}$. This model was parameterised in terms of CL, V, $K_{\mathrm{a}}$, LAG and $D$. Both of these models allowed all pharmacokinetic parameters to vary between individuals according to an exponential error model; both were fitted using ADVAN2.

The relationships between pharmacokinetic parameters (apparent clearance, AUC and apparent volume of distribution), peak concentrations of 13-cisRA and 4-oxo-13-cisRA on days 1 and 14 of treatment, and covariates were examined graphically and either $t$-tests or linear regression applied as appropriate. The pharmacokinetic parameter estimates used were the empirical Bayes estimates obtained from the modified zero-order absorption model, except the peak concentrations. Covariates investigated were age, weight, ALT, bilirubin, creatinine, GFR, sex, method of administration and CTC grade $3 / 4$ toxicity. The logarithm of all pharmacokinetic parameters and continuous covariates was used in preference to the untransformed variables owing to the skewed nature of the data. Empirical Bayes estimates of pharmacokinetic parameters for courses 4 and 6 , where data were available, were obtained from the final population model.

The Cox proportional hazards model was used to investigate any relationship between pharmacokinetic end points, including peak levels of 13-cisRA and 4-oxo-13-cisRA on days 1 and 14 on the first available course of treatment, and time to relapse/ progression of disease, relative to the first 13-cisRA administration.

\section{RESULTS}

\section{Patient characteristics and treatment}

Twenty-nine children were entered into the study. One patient had to withdraw from the study before samples being taken, owing to removal of their central venous catheter. The study population had a median age of 3.2 years (range 1.1-18.7) and included 16 male and 12 female patients. Patient characteristics for the 28 evaluable patients are given in Table 1.

\section{Pharmacokinetics}

The pharmacokinetics of 13-cisRA were best described by a modified one-compartment, zero-order absorption model combined with an absorption lag time (LAG) allowing a full covariance 
matrix for the random effects. The differential equations for the final model are:

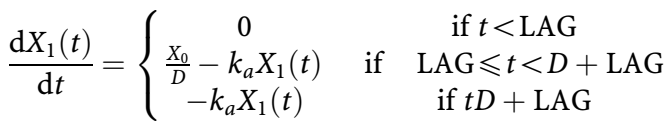

$$
\begin{aligned}
& \frac{\mathrm{d} X_{2}(t)}{\mathrm{d} t}=k_{a} \cdot X_{1}(t)-\frac{\mathrm{CL}}{V} \cdot X_{2}(t),
\end{aligned}
$$

where $X_{0}$ is the dose of 13cisRA administered at time $t=0$ and assumed to enter the system at time $t=\mathrm{LAG} ; X_{1}(t)$ is the amount of drug in the dose compartment at time $t ; X_{2}(t)$ is the amount of drug in the observation compartment at time $t ; D$ is the duration of the zero-order input; $k_{\mathrm{a}}$ is the rate of the first-order absorption; CL is apparent clearance and $V$ is apparent volume of distribution.

However, both the transit model and the first-order absorption model with lag time gave very similar parameter estimates and individual fits, despite having slightly larger objective function values (Table 2). Mean population pharmacokinetic parameters were; apparent clearance $15.91 \mathrm{~h}^{-1}$, apparent volume of distribution 851 and absorption lag time $40 \mathrm{~min}$. There was large interindividual variability associated with these parameters. Clearance had an inter-individual CV of $69 \%$, while both volume of distribution and $K_{\mathrm{a}}$ had CVs in excess of $100 \%$. Correction of clearance or volume of distribution for body surface area did not reduce the $\mathrm{CV}$ (Table 3 ).

Table I Patient characteristics

\begin{tabular}{lcr}
\hline Characteristic & No. of patients & $\%$ \\
\hline Age (years) & 8 & \\
$<3$ & 13 & 29 \\
$3-5$ & 4 & 46 \\
$6-10$ & 3 & 14 \\
$11-18$ & & 11 \\
Sex & 16 & \\
Male & 12 & 57 \\
Female & & 43 \\
& & \\
Weight (kg) & 1 & 3 \\
$<10$ & 21 & 75 \\
I0-20 & 3 & 11 \\
$20-30$ & 3 & 11 \\
$>30$ & & \\
& & 7 \\
Disease stage & 2 & \\
3 (with MYCN amplification) & 26 & \\
4 (age $>$ I year) & & 54 \\
MYCN amplification & 15 & 46 \\
Yes & 13 & \\
No & & \\
\hline
\end{tabular}

There was a large degree of variation between courses in day 1 13-cisRA levels (Figure 1; Tables 3 and 4). Whereas some patients showed little variation between courses (e.g. patients 15 and 20), others exhibited large differences (e.g. patients 2 and 7). In contrast, variation in 13-cisRA levels between study days (1 and 14) of the same course, in particular course 2, was relatively small (Figure 2A).

Owing to difficulties in obtaining stable parameter estimates, the inclusion of covariates into a population pharmacokinetic model was not undertaken. Instead, the relationship between pharmacokinetic parameter estimates and covariates was examined via plots and either $t$-tests or linear regression as appropriate. 13-cisretinoic acid AUC and day 14 peak 4-oxo-13-cisRA levels were both found to be related to weight, age and whether or not the capsule had been opened before administration. Higher weight and age were also associated with larger AUCs and peak 4-oxo13-cisRA levels $(P<0.02$ in all cases). Day 1 peak 13-cisRA concentrations were found to be linked to method of administration $(P<0.02)$. When 13-cisRA capsules were swallowed, AUC was found to be on average 1.95-fold larger ( $95 \%$ confidence interval (CI) $1.16,3.28)$ than when capsules were opened; day 1 peak $13-$ cisRA levels were 2.44-fold higher (CI 1.23, 4.83) and day 14 peak 4-oxo-13-cisRA levels were 2.65-fold higher (CI 1.41, 5.00) when capsules were unopened. Figure 3 shows AUC values for 13-cisRA

Table 3 Summary statistics for empirical Bayes pharmacokinetic parameter estimates on study day I, analysed by course

\begin{tabular}{|c|c|c|c|c|c|c|c|}
\hline Parameter & Course & $\mathbf{N}$ & Mean & s.d. & Min & Median & Max \\
\hline \multirow[t]{3}{*}{$\mathrm{CL} / \mathrm{F}\left(\mathrm{Ih}^{-1}\right)$} & 2 & 26 & 20.7 & 16.7 & 4.8 & 17.8 & 80.8 \\
\hline & 4 & 12 & 12.5 & 6.7 & 3.4 & 11.1 & 24.7 \\
\hline & 6 & 10 & 13.2 & 6.9 & 7.1 & 10.9 & 30.7 \\
\hline \multirow[t]{3}{*}{ V/F (I) } & 2 & 26 & 121.1 & 128.7 & 21.6 & 75.0 & 490.2 \\
\hline & 4 & 12 & 40.9 & 22.1 & 13.2 & 38.2 & 100.3 \\
\hline & 6 & 10 & 68.8 & 57.2 & 30.1 & 41.4 & 194.4 \\
\hline \multirow[t]{3}{*}{$\mathrm{A} \cup \mathrm{C}\left(\mu \mathrm{molhl^{-1 }}\right)$} & 2 & 26 & 13.2 & 9.0 & 2.1 & 10.5 & 37.3 \\
\hline & 4 & 12 & 20.8 & 11.0 & 6.1 & 20.2 & 43.7 \\
\hline & 6 & 10 & 17.2 & 8.1 & 5.4 & 15.8 & 31.3 \\
\hline \multirow[t]{3}{*}{$\mathrm{CL} / \mathrm{F}\left(\mathrm{Ih}^{-1} \mathrm{~m}^{-2}\right)$} & 2 & 26 & 31.9 & 27.2 & 7.7 & 24.0 & 136.2 \\
\hline & 4 & 12 & 17.7 & 11.4 & 5.9 & 13.4 & 41.0 \\
\hline & 6 & 10 & 19.1 & 12.4 & 7.6 & 15.8 & 51.2 \\
\hline \multirow[t]{3}{*}{$V / F\left(\mid m^{-2}\right)$} & 2 & 26 & 177.4 & 173.7 & 29.7 & 125.7 & 662.7 \\
\hline & 4 & 12 & 58.8 & 39.7 & 21.7 & 51.3 & 168.3 \\
\hline & 6 & 10 & 100.5 & 94.9 & 34.0 & 55.6 & 324.5 \\
\hline \multirow[t]{3}{*}{ LAG (min) } & 2 & 26 & 41.2 & 10.6 & 23.7 & 39.0 & 57.7 \\
\hline & 4 & 12 & 49.4 & 7.8 & 37.8 & 52.5 & 62.5 \\
\hline & 6 & 10 & 43.7 & 7.5 & 31.9 & 44.1 & 54.5 \\
\hline
\end{tabular}

CL/F, apparent clearance; LAG, absorption lag time; AUC, area under the curve, extrapolated to infinity; $N$, number of patients studied on each course of treatment. CL/F and VIF also given as values corrected for body surface area. Course 2 refers to first available course. This was course $I$ in 5 patients and course 3 in one patient.

Table 2 Comparison of population pharmacokinetic parameter estimates for various models; coefficient of variation (\%) shown in paranthesis

\begin{tabular}{|c|c|c|c|c|c|c|c|}
\hline Model & OFV & CL/F & $\mathbf{K a}$ & V/F & LAG/MTT & $\mathbf{N N}$ & D \\
\hline First-order absorption with lag time & 24.3 & $15.40(63.8)$ & $0.050(236)$ & $64.07(102)$ & $53.52(11.7)$ & - & - \\
\hline
\end{tabular}

OFV, objective function value; $\mathrm{CL} / \mathrm{F}$, apparent clearance $\left(\mathrm{I}^{-1}\right) ; \mathrm{K}_{\mathrm{a}}$, absorption rate $\left(\mathrm{I} \mathrm{min}^{-1}\right)$; V/F, apparent volume of distribution (I); LAG, absorption lag time (min); MTT, mean time to absorption transition (min); NN, number of transit compartments; $D$, absorption duration (min). 
Opened and mixed with food
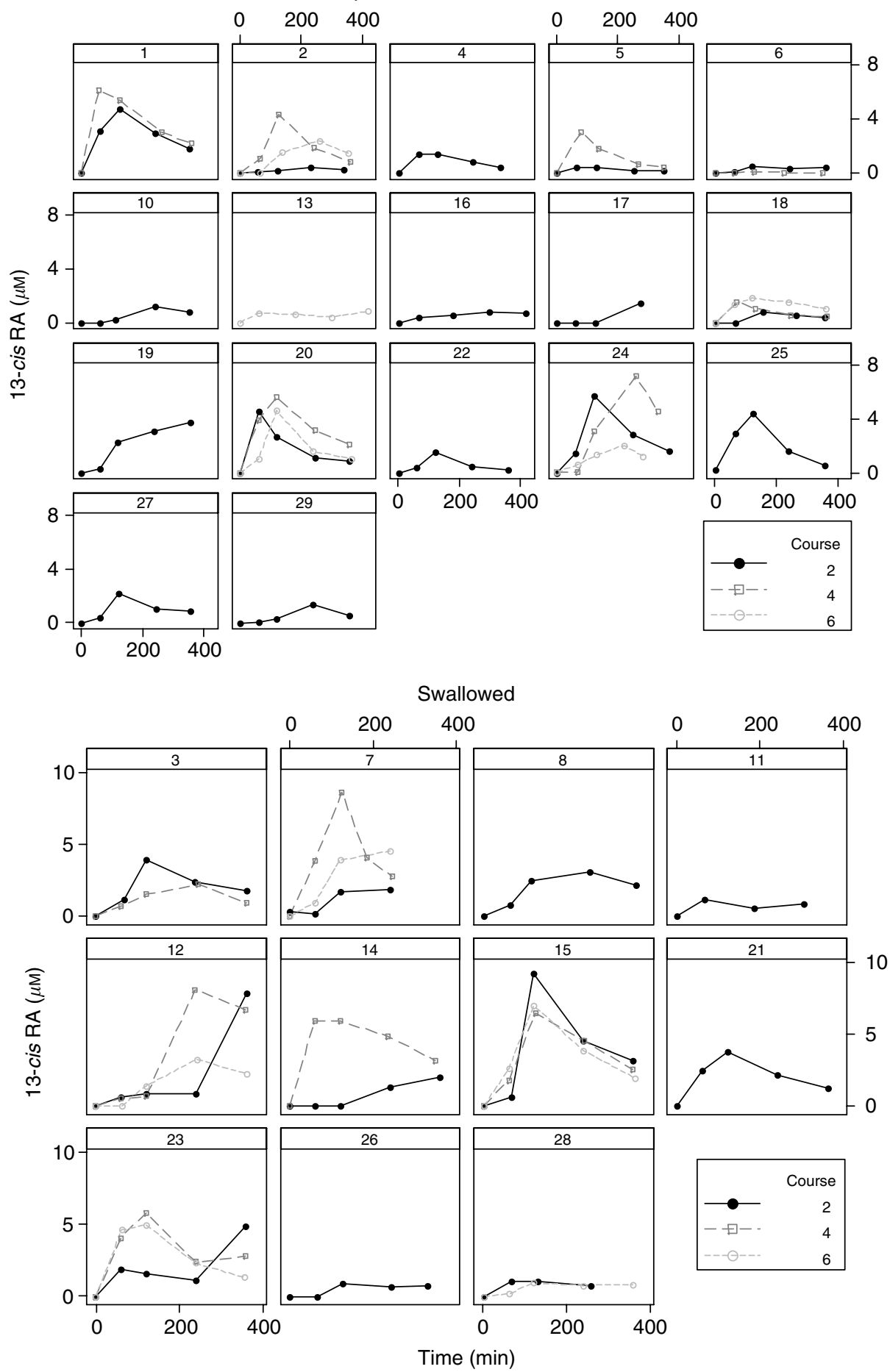

Figure I 13-cis-retinoic acid data; study day I for all patients shown by course; separate graphs shown by method of administration. Patient 26 received I3cisRA via an NG tube.

observed on day 1 of course 2 of treatment in patients who swallowed 13-cisRA capsules $v s$ patients for whom the capsule contents were mixed with food.

Larger absolute doses of 13-cisRA were associated with higher day 14 peak 4-oxo-13-cisRA levels $(P=0.01)$. A larger 13 -cisRA apparent volume of distribution was associated with higher creatinine levels $(P=0.02)$, but none of the covariates were found to be related to apparent clearance of 13-cisRA, peak 13-cisRA levels from day 14 or peak 4-oxo-13-cisRA levels from day 1.

\section{Oxidative metabolism}

Extensive accumulation of 4-oxo-13-cisRA occurred in all patients during each treatment course, with plasma concentrations higher than those of 13-cisRA on day 14 of course 2 of treatment in 16 out of 23 patients for whom data were available (Figure 4; Table 4). In course 2, peak concentrations of 4-oxo-13-cisRA increased from 0.2 to $5.9 \mu \mathrm{m}$ on day 1 to $0.7-11.6 \mu \mathrm{m}$ on day 14 of treatment, as compared with 13-cisRA plasma concentrations of $0.3-5.5 \mu \mathrm{M}$ 
on day 14. Similar increases in the level of metabolite over the 14 days of treatment were observed in subsequent courses (Figure 4). No other retinoic acid metabolites were detected in plasma samples of patients receiving 13-cisRA and concentrations of ATRA accounted for less than $5 \%$ of total retinoids in all samples.

\section{Clinical response and toxicity}

Of the 28 evaluable patients, 13 (46\%) were alive with no disease at follow-up. Time to follow-up ranged from 18 months to 4 years. Of the remaining patients, three $(11 \%)$ were alive with disease progression and $12(43 \%)$ had died following disease relapse.
There was a greater likelihood of relapse for patients with higher day 14 peak 4-oxo-13cisRA plasma concentrations $(P=0.014$; Cox regression analysis). No other pharmacokinetic parameters were related to time to relapse or survival.

Treatment was reasonably well tolerated, although several patients had persistent haematological toxicity following the previous myeloablative therapy. Nine patients experienced some form of mild skin toxicity (eight CTC grade 2, one grade 3), with only one report of mild cheilitis. Hypercalcaemia (grade 2 or 3 ) was reported in two patients. There was no evidence to suggest that any of the toxicities observed were linked to the pharmacokinetics of 13-cisRA or its metabolite.
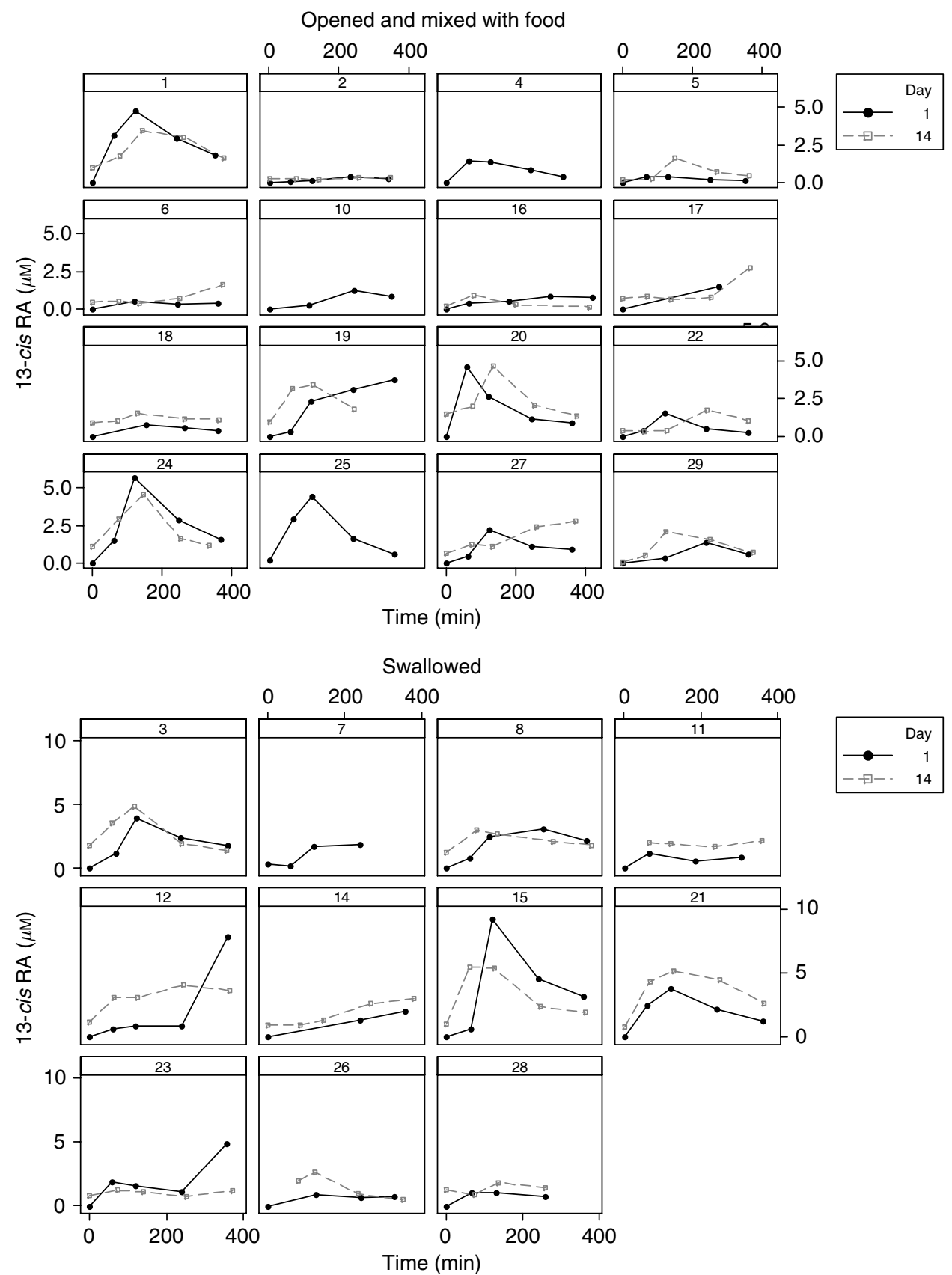

Figure 2 13-cis-retinoic acid data; course 2 for all patients shown by study day; separate graphs shown by method of administration. Patient 26 received 13 cisRA via an NG tube. 
Table 4 Summary statistics for 13-cisRA Cmax and 4-oxo-13-cisRA Cmax on study day I and I4, analysed by course

\begin{tabular}{lcccc}
\hline Parameter & Course & N & Mean & s.d. \\
\hline |3-cisRA & 2 & & & \\
Day | $(\mu \mathrm{M})$ & 4 & 27 & 2.78 & 2.31 \\
& 6 & 13 & 5.43 & 2.23 \\
& 2 & 10 & 3.25 & 1.99 \\
Day |4 $(\mu \mathrm{M})$ & 4 & & & \\
& 6 & 13 & 2.83 & 1.44 \\
& & 10 & 4.71 & 2.40 \\
4-oxo- |3-cisRA & 2 & & 2.52 & 1.85 \\
Day | $(\mu \mathrm{M})$ & 4 & 27 & & \\
& 6 & 13 & 1.30 & 1.21 \\
Day |4 $(\mu \mathrm{M})$ & 2 & 10 & 1.31 & 0.85 \\
& 4 & 27 & 4.67 & 3.17 \\
& 6 & 13 & 6.43 & 4.11 \\
& & 10 & 3.49 & 1.98 \\
\hline
\end{tabular}

Abbrevitions: $\mathrm{RA}=$ retinoic acid; s.d. = standard deviation

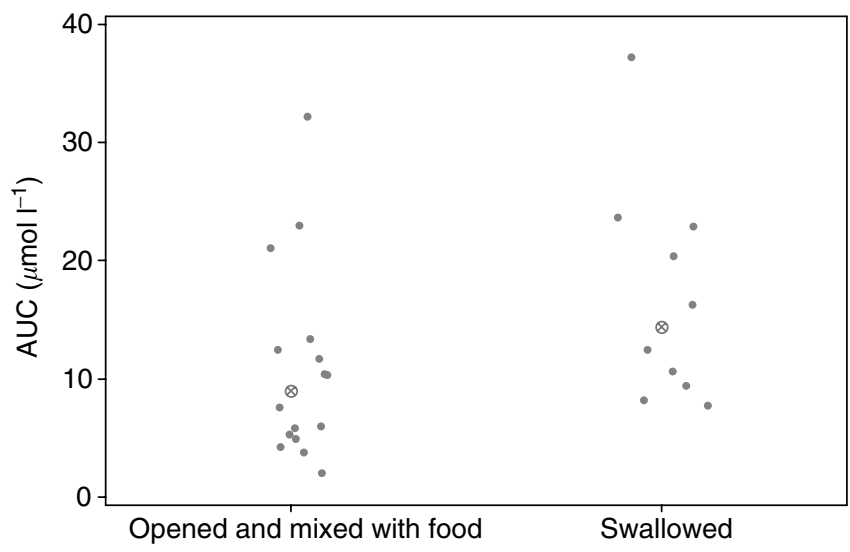

Figure 3 Area under the curve of | 3-cisRA observed on day I of course 2 of treatment in patients who swallowed I3-cisRA capsules vs those patients for whom the capsules were opened and the contents mixed with food before administration. Median values are identified with a cross.

\section{DISCUSSION}

The addition of 13-cisRA to the treatment of high-risk neuroblastoma has been shown to improve 3-year event-free survival rates. Nevertheless, despite the addition of 13-cisRA to high-dose myeloablative chemotherapy treatment protocols, more than half of patients still suffer relapse within 3 years. While in some patients relapse is mostly influenced by the biology of the tumour, the high degree of variability in the pharmacokinetics and metabolism of 13-cisRA observed in this study suggests that there is scope for further improvements based on individualisation of dosing or schedules.

A series of population pharmacokinetic models were fitted to 13-cisRA plasma concentration-time data from 28 children with high-risk neuroblastoma. Following detailed analysis of these data, the pharmacokinetics of 13-cisRA were best described by a modified one-compartment, zero-order absorption model combined with an absorption lag time. Some patients had almost no absorption lag time followed by a very rapid absorption, whereas in other patients a prolonged lag time was observed, together with very slow absorption. Conversely, a very delayed but rapid rise in plasma concentrations was seen in some patients. Thus, a relatively complex model with an absorption lag time was required for all of the standard models. However, the simple first- and zero-order models did not fit particularly well when this approach was taken. Use of the modified zero-order model or the transit model provided a much better fit to the data.

The dosing regimen of $80 \mathrm{mg} \mathrm{m}^{-2}$ twice daily for 14 days was associated with significant inter-patient variation in 13-cisRA pharmacokinetics, comparable with that observed following ATRA administration for the treatment of APL (Lanvers et al, 2003). For 13-cisRA, pharmacokinetics may be influenced by a number of factors, including both the method of drug administration and the extent of metabolism.

Children diagnosed with high-risk neuroblastoma are commonly aged between 1 and 5 years, presenting a practical problem with regards to administering large numbers of 13-cisRA capsules. For example, an average child of 5 years with a surface area of $0.75 \mathrm{~m}^{2}$ would require a daily dose of $120 \mathrm{mg}$, administered as six large $20 \mathrm{mg}$ capsules or $245 \mathrm{mg}$ capsules. Younger children are therefore often unable to take 13-cisRA unless it is removed from the capsules before administration. In addition to the safety concerns that may arise from this practice, with regards to the handling of a potentially teratogenic substance, opening the capsule may impact on the actual dose of drug received. This may reflect loss of drug during handling in addition to the welldocumented instability of 13-cisRA in the presence of light.

Of the 28 patients recruited to the current study, 17 (age 1.110.7 years) required the capsules to be opened and the contents mixed with food. Plasma concentrations of 13-cisRA in these patients were significantly lower than those observed in children who were able to swallow the capsules, although both groups exhibited a wide variation in plasma concentrations. No attempt was made to control food intake around the time of administration. A previous study has reported higher plasma concentrations when 13-cisRA was administered within $1 \mathrm{~h}$ before or after a standard meal, compared with the fasted state (Colburn et al, 1983). Application of the data presented here to guide dosing of 13-cisRA would also need to be adapted to reflect differences in the administration of 13-cisRA in different countries, for example punching a hole in the capsule so that it can be chewed before swallowing. The younger children, for whom the capsules were most likely to have been opened, also received the lowest absolute doses in milligrams.

Following oral administration, 13-cisRA may be subject to firstpass metabolism and subsequent plasma concentrations will depend on the rate and extent of metabolism to 4-oxo-13-cisRA, generally thought to represent a pathway of retinoid inactivation. Oxidative metabolism has previously been observed in studies with ATRA (Muindi et al, 1992; Rigas et al, 1993) and fenretinide (Villani et al, 2004), and auto-induction of oxidative metabolism has been associated with disease relapse, following chronic administration of ATRA in APL (Muindi et al, 1992). This can be avoided by the use of intermittent ATRA dosing schedules (Adamson et al, 1995), an approach that has also been recommended to minimise the side effects of ATRA in children (Lanvers et al, 2003). Our results with 13-cisRA show an accumulation of the 4-oxo-metabolite between days 1 and 14 of treatment in all patients, with metabolite concentrations higher than those of the parent drug by day 14 in approximately $70 \%$ of patients. There was no corresponding decrease in parent drug concentrations, which would have indicated enzyme induction. Thus, repeated dosing of 13 cisRA is unlikely to be associated with cytochrome P450 (CYP) enzyme induction, as is seen with ATRA (Adamson et al, 1993).

With the limited size of the current study, it is difficult to obtain a clear indication of the impact of inter-patient pharmacokinetic variation and metabolism of 13-cisRA on clinical efficacy or toxicity. The degree of toxicity was similar to that reported in the Phase I study of 13-cisRA at the equivalent dose level (Villablanca 

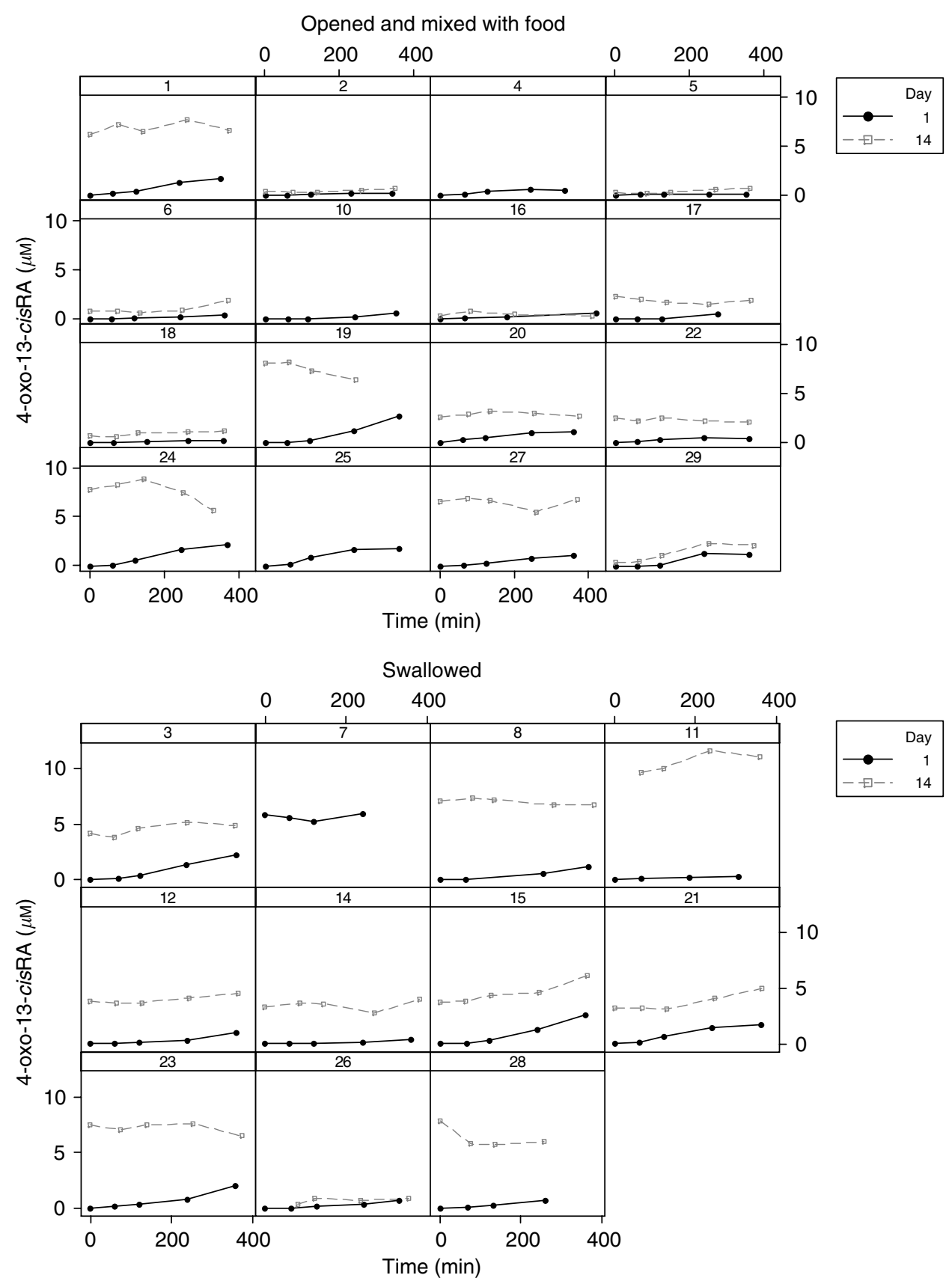

Figure 4 4-oxo-I3-cisRA data; course 2 for all patients shown by study day; separate graphs shown by method of administration. Patient 26 received 13 cisRA via and NG tube.

et al, 1995). A statistically significant relationship was observed between the incidence of disease relapse and plasma metabolite concentrations, with an increased likelihood of relapse for patients with higher day 14 peak 4-oxo-13-cisRA levels. A corresponding decrease in parent drug was not detectable. It should be noted that older patients, who may have a higher risk of relapse, would receive higher doses of unopened capsules.

As lower doses of retinoic acid have been shown to be ineffective in the treatment of neuroblastoma (Matthay et al, 1999; Kohler et al, 2000), the extent of inter-patient pharmacokinetic variation following high-dose 13-cisRA therapy warrants further investigation. Individuals with plasma concentrations below those associated with antitumour activity may require an increased dose. One approach to overcome interindividual variation, and to ensure that plasma concentrations are not compromised by the method of administration, would be to monitor plasma concentrations on day 1 of treatment. Data in Figure 2 indicate that plasma concentrations do not vary substantially between days of treatment, thus dose adjustment based on the day 1 plasma concentrations is feasible.

It is notable that the plasma concentrations achieved in the current study are up to four-fold lower than those reported in a phase I study of the same formulation (Khan et al, 1996). The reasons for this difference are not easily identified. The age range, and thus the doses administered, in the phase I study population are not different to that studied here. It may be that the method of administration was different in the previous study, although the difference in AUC is equally marked in our patients who swallowed 
the capsules whole. There are differences in the assay for drug and metabolite in plasma, but our method was stringently validated and rigorous efforts were employed to minimise loss of drug due to photodegradation. Data on ATRA and the 4-oxo metabolite are comparable between the two studies, although little detail is given in the phase I report (Khan et al, 1996).

The conversion to 4-oxo-13-cisRA is the major route of 13-cis RA metabolism. Although there was no indication that this led to significantly lower parent retinoid levels during treatment, higher 13-cisRA levels may be achieved by limiting the extent of metabolism. Although oxidation of 13-cisRA may be mediated by a number of enzymes (Sonneveld et al, 1998; Nadin and Murray, 1999; Chen et al, 2000), specific inhibitors of retinoid metabolism, such as R116010, may be used to inhibit such oxidation (Armstrong et al, 2005) and thus may increase 13-cisRA plasma concentrations. These data also indicate that lower systemic 13cisRA exposures are associated with the practice of opening the capsules and mixing the contents with food before administration. This finding highlights the importance of obtaining appropriate pharmaceutical formulations of medicines for children. Although a simple increase in 13-cisRA dose is likely to overcome this

\section{REFERENCES}

Adamson PC, Bailey J, Pluda J, Poplack DG, Bauza S, Murphy RF, Yarchoan $\mathrm{R}$, Balis FM (1995) Pharmacokinetics of all-trans-retinoic acid administered on an intermittent schedule. J Clin Oncol 13: 1238-1241

Adamson PC, Boylan JF, Balis FM, Murphy RF, Godwin KA, Gudas LJ, Poplack DG (1993) Time course of induction of metabolism of all-transretinoic acid and the upregulation of cellular retinoic acid-binding protein. Cancer Res 53: $472-476$

Armstrong JL, Ruiz M, Boddy AV, Redfern CPF, Pearson ADJ, Veal GJ (2005) Increasing the intracellular availability of all-trans retinoic acid in neuroblastoma cells. Brit J Cancer 92: 696-704

Beal S, Sheiner L (1989) NONMEM Users Guide - Part I Users Basic Guide. San Francisco, CA: NONMEM Project Group, UCSF

Chen H, Fantel AG, Juchau MR (2000) Catalysis of the 4-hydroxylation of retinoic acids by CYP3A7 in human fetal hepatic tissues. Drug Metab Dispos 28: $1051-1057$

Colburn WA, Gibson DM, Wiens RE, Hanigan JJ (1983) Food increases the bioavailability of isotretinoin. J Clin Pharmacol 23: 534-539

Cole M, Price L, Parry A, Keir MJ, Pearson ADJ, Boddy AV, Veal GJ (2004) Estimation of glomerular filtration rate in paediatric cancer patients using (CR)-C-51-EDTA population pharmacokinetics. Brit J Cancer 90: $60-64$

Finklestein JZ, Krailo MD, Lenarsky C, Ladisch S, Blair GK, Reynolds CP, Sitarz AL, Hammond GD (1992) 13-cis-retinoic acid (Nsc-122758) in the treatment of children with metastatic neuroblastoma unresponsive to conventional chemotherapy - report from the Childrens-Cancer-StudyGroup. Med Pediatr Oncol 20: 307-311

Khan AA, Villablanca JG, Reynolds CP, Avramis VI (1996) Pharmacokinetic studies of 13-cis-retinoic add in pediatric patients with neuroblastoma following bone marrow transplantation. Cancer Chemother Pharmacol 39: 34-41

Kohler JA, Imeson J, Ellershaw C, Lie SO (2000) A randomized trial of 13cis retinoic acid in children with advanced neuroblastoma after highdose therapy. Brit J Cancer 83: $1124-1127$

Lanvers C, Reinhardt D, Dubbers A, Wagner-Bohn A, Creutzig U, Ritter J, Boos J (2003) Pharmacology of all-trans-retinoic acid in children with acute promyelocytic leukemia. Med Pediatr Oncol 40: 293-301

Matthay KK, Reynolds CP (2000) Is there a role for retinoids to treat minimal residual disease in neuroblastoma? Brit J Cancer 83: 1121-1123

Matthay KK, Villablanca JG, Seeger RC, Stram DO, Harris RE, Ramsay NK, Swift P, Shimada H, Black CT, Brodeur GM, Gerbing RB, Reynolds CP (1999) Treatment of high-risk neuroblastoma with intensive chemotherapy, radiotherapy, autologous bone marrow transplantation and 13-cisretinoic acid. New Engl J Med 341: 1165-1173 problem, it may be more appropriate to design a study incorporating the monitoring of plasma concentrations, with a view to standardisation of drug exposure and the development of more robust pharmacokinetic-pharmacodynamic relationships. The data presented here show that optimisation of dosing of 13cisRA in high-risk neuroblastoma is possible, based on knowledge of the clinical pharmacology of this drug.

\section{ACKNOWLEDGEMENTS}

We thank the patients, research nurses and clinicians who participated in the study at the following UKCCSG centres: Royal Victoria Infirmary, Newcastle upon Tyne; Great Ormond Street Hospital, London; Manchester Children's Hospital; St James's Hospital, Leeds; Royal Liverpool Children's Hospital; Royal Marsden Hospital, Surrey; Addenbrooke's Hospital, Cambridge; Bristol Royal Hospital for Children, Bristol; Southampton General Hospital. This research was supported by Cancer Research UK and by the North of England Children's Cancer Research Fund.
Muindi J, Frankel SR, Miller WH, Jakubowski A, Scheinberg DA, Young CW, Dmitrovsky E, Warrell RP (1992) Continuous treatment with alltrans retinoic acid causes a progressive reduction in plasma drug concentrations - implications for relapse and retinoid resistance in patients with acute promyelocytic leukemia. Blood 79: 299-303

Nadin L, Murray M (1999) Participation of CYP2C8 in retinoic acid 4hydroxylation in human hepatic microsomes. Biochem Pharmacol 58: $1201-1208$

Reynolds CP, Kane DJ, Einhorn PA, Matthay KK, Crouse VL, Wilbur JR, Shurin SB, Seeger RC (1991) Response of neuroblastoma to retinoic acid in vitro and in vivo. In Advances in Neuroblastoma Research Evans AE, Dangio GJ, Knudson AG, and Seeger RC (eds). Vol 366, pp 203-211; Progress in Clinical and Biological Research, Wiley-Liss: New york

Rigas JR, Francis PA, Muindi JRF, Kris MG, Huselton C, Degrazia F, Orazem JP, Young CW, Warrell RP (1993) Constitutive variability in the pharmacokinetics of the natural retinoid, all-trans-retinoic acid, and its modulation by ketoconazole. J Natl Cancer I 85: $1921-1926$

Savic R, Jonker D, Kerbusch T, Karlsson M (2004) Evaluation of a Transit Compartment Model Versus a Lag Time Model for Describing Drug Absorption Delay. Population Approach Group Europe: Uppsala, Sweden, pp 513

Shah VP, Midha KK, Dighe S, McGilveray IJ, Skelly JP, Yacobi A, Layloff T, Viswanathan CT, Cook CE, McDowall RD, Pittman KA, Spector S (1992) Analytical methods validation - bioavailability, bioequivalence, and pharmacokinetic studies. J Pharm Sci 81: 309-312

Sonneveld E, van den Brink CE, van der Leede BJM, Schulkes RKAM, Petkovich M, van der Burg B, van der Saag PT (1998) Human retionic acid (RA) 4-hydroxylase (CYP26) is highly specific for all-trans-RA and can be induced through RA receptors in human breast and colon carcinoma cells. Cell Growth Differ 9: 629-637

Veal GJ, Errington J, Redfern CPF, Pearson ADJ, Boddy AV (2002) Influence of isomerisation on the growth inhibitory effects and cellular activity of 13-cis and all-trans retinoic acid in neuroblastoma cells. Biochem Pharmacol 63: 207-215

Villablanca JG, Khan AA, Avramis VI, Seeger RC, Matthay KK, Ramsay NK, Reynolds CP (1995) Phase I trial of 12-cis-retinoic acid in children with neuroblastoma following bone marrow transplantation. J Clin Oncol 13: $894-901$

Villani MG, Appierto V, Cavadini E, Valsecchi M, Sonnino S, Curley RW, Formelli $F$ (2004) Identification of the fenretinide metabolite 4-oxofenretinide present in human plasma and formed in human ovarian carcinoma cells through induction of cytochrome P450 26A1. Clin Cancer Res 10: 6265-6275 